\title{
Improved measurement of the shape of the electron
}

\author{
J. J. Hudson, D. M. Kara, I. J. Smallman, B. E. Sauer, M. R. Tarbutt, E. A. Hinds \\ Centre for Cold Matter, Blackett Laboratory, \\ Imperial College London, Prince Consort Road, \\ London SW7 2AZ, United Kingdom.
}

April 7, 2011

The electron is predicted to be slightly aspheric [1], with a distorsion characterised by the electric dipole moment (EDM), $d_{e}$. No experiment has ever detected this deviation. The standard model of particle physics predicts that $d_{e}$ is far too small to detect [2], being some eleven orders of magnitude smaller than the current experimental sensitivity. However, many extensions to the standard model naturally predict much larger values of $d_{e}$ that should be detectable [3]. This makes the search for the electron EDM a powerful way to search for new physics and constrain the possible extensions. In particular, the popular idea that new supersymmetric particles may exist at masses of a few hundred $\mathrm{GeV}$ is difficult to reconcile with the absence of an electron EDM at the present limit of sensitivity[4, 2]. The size of the EDM is also intimately related to one 
of the largest outstanding problems in cosmology: why does our universe have so little antimatter? One solution is to propose some undiscovered particle interaction that breaks the symmetry between matter and antimatter [5]. Our experiment constrains such interactions since they also generate a measurable EDM in most models of particle physics [2]. We use cold, polar molecules to measure the electron EDM, obtaining the result $d_{e}=\left(-2.4 \pm 5.7_{\text {stat }} \pm 1.5_{\text {syst }}\right) \times 10^{-28} e \cdot \mathbf{c m}$, which sets a new upper limit of $\left|d_{e}\right|<10.5 \times 10^{-28} e \cdot \mathbf{c m}$ with $90 \%$ confidence. Our result, consistent with zero, indicates that the electron is spherical at this improved level of precision. Our measurement, of atto-eV energy shifts in a molecule, probes new physics at the tera-eV energy scale [2], thus providing important input for theories of particle physics and cosmology.

Just as a magnetic dipole moment $\boldsymbol{\mu}$ in a magnetic field $\mathbf{B}$ has an energy $-\boldsymbol{\mu} \cdot \mathbf{B}$, an electric dipole moment $\mathbf{d}$ in an electric field $\mathbf{E}$ has an energy $-\mathbf{d} \cdot \mathbf{E}$ in the non-relativistic limit. A permanent EDM of the electron must lie along its spin [6], $\mathbf{d}=d_{e} \boldsymbol{\sigma}$, making the electron's energy depend on whether the spin is parallel or antiparallel to E. In an atom or molecule with an unpaired valence electron, the interaction of the electron EDM with an applied electric field results in an energy difference between two states that differ only in their spin orientation. This energy difference is proportional to $d_{e}$ and changes sign when the direction of the field is reversed. A sensitive method for measuring this energy difference is to align the spin perpendicular to the field and measure its precession rate, which is proportional to the energy difference. An alternative description of the method is in terms of an interferometer. There is quantum interference between the two spin states, and the EDM appears as 
an interferometer phase shift that changes sign when the electric field is reversed.

In order to improve on the previous limit [7] we have developed a technique using the dipolar molecule $\mathrm{YbF}[8]$ instead of the spherical Tl atom. This brings two great advantages. First, at our operating field the interaction energy $[9,10,11,12,13,14,15]$ of $\mathrm{YbF}$ due to $d_{e}$ is 220 times larger than that obtained using Tl in a large field [7]. Second, the motional magnetic field, a source of systematic error which plagued the Tl experiment, has negligible effect on $\mathrm{YbF}$ [8]. Because of these advantages, it is possible to improve over the $\mathrm{Tl}$ experiment using YbF molecules, even though the molecules are produced in much smaller numbers. A number of other EDM measurements, based on electron spin precession in atoms, molecules, molecular ions, or solids, are currently ongoing [4].

Figure 1 shows the interferometer apparatus [16]. Pulses of YbF molecules are emitted by the source [17]. The experiment uses those molecules in the $F=0$ and $F=1$ hyperfine levels of the ground state. The molecules pass through a first fluorescence detector, the pump detector, which simultaneously measures and empties out the $F=1$ population. Then they enter a pair of electric field plates, between which are static electric and magnetic fields $(E, B) \hat{z}$. This region is magnetically shielded. An rf pulse is applied to transfer molecules from $\left|F, m_{F}\right\rangle=|0,0\rangle$ to the state $\frac{1}{\sqrt{2}}(|1,+1\rangle+|1,-1\rangle)$. The molecules then evolve freely for a time $T$, during which the $m_{F}= \pm 1$ components develop a phase difference of $2 \phi=$ $2\left(\mu_{B} B-d_{e} E_{\text {eff }}\right) T / \hbar$. This is due to the Zeeman shift $+\mu_{B} B m_{F}$ [18] and to the EDM shift expressed by the effective interaction $-d_{e} E_{\text {eff }} m_{F}$ (see Methods). A second rf pulse is then applied, resulting in a final $F=0$ population proportional to $\cos ^{2} \phi$ which the second 
fluorescence detector subsequently measures. For every pulse of molecules, the time-resolved signals from the pump and probe detectors are recorded; an example probe signal is shown in figure 2.

Scanning the phase difference via the magnetic field generates an interference curve, shown in figure 3. Reversal of the applied electric field produces a small phase shift $\delta \phi=$ $2 d_{e} E_{\text {eff }} T / \hbar$, leading to a change in the detector count $\delta I=\frac{d I}{d \phi} \delta \phi$. This is maximized by operating the interferometer at $B= \pm 13.6 \mathrm{nT}$ which corresponds to $\phi= \pm \pi / 4$, the steepest points on either side of the central fringe (figure 3). The intensity change is opposite on the two sides of the fringe because the slopes are opposite. Thus the EDM signal $\delta I$ is the part of the fluorescence count that is correlated with the sign of $E \cdot B$. We calibrate the slope $\frac{d I}{d \phi}$ by making a step $\delta B= \pm 1.7 \mathrm{nT}$ in magnetic field magnitude, and this too is done on each side of the central fringe. In addition to $E, B$ and $\delta B$, several other parameters are switched in the experiment. The laser frequency (LF) is stepped by $\pm 340 \mathrm{kHz}$, the frequencies of the two rf pulses (RF1F and RF2F) are independently stepped by $\pm 1.5 \mathrm{kHz}$, their amplitudes (RF1A and RF2A) are independently stepped by $\pm 5 \%$, and the phase difference (RF $\Phi$ ) between them is stepped around a randomly chosen value, $\phi_{0}$, by $\pm \pi / 2$. A computer places the machine in a new switch state before every beam pulse. The measurements are grouped into 'blocks' of 4096 beam pulses, over which all 512 combinations of switch states are covered equally. Error signals, derived from each block of data, are used to servo the switched parameters to switch around their optima.

Our measurement is derived from 6194 blocks of data taken in 2010, comprising 25 mil- 
lion molecular beam pulses, together with many subsidiary measurements used to search for systematic errors. To analyze the data, we select the central $130 \mu$ s of each probe pulse (figure 2) and normalize it pulse by pulse to the pump fluorescence. This minimises the effect of fluctuations of the molecular beam intensity. We calculate how much of the gated, normalized fluorescence signal is correlated with all 512 possible combinations of the modulated parameters. These correlations are called 'channels' and are denoted by $\{X\}, X$ indicating the parameter (or parameter combination) being modulated. The EDM phase shift, normalised to the shift from the small magnetic field step $\delta B$, is $\{E \cdot B\} /\{\delta B\}$. The other channels are valuable in elucidating the operation of the apparatus. Throughout the investigation the EDM values were concealed by adding a fixed unknown offset, which was only removed once the data collection and analysis were complete.

The EDM values obtained from the set of blocks are almost normally distributed but there tend to be a few more points in the wings of the distribution than in a normal distribution. The same is true of other quantities of interest that we extract from the data. For all these quantities, we calculate the 5\% trimmed mean [19], a simple robust statistic that drops the largest and smallest $5 \%$ of the data. We use the bootstrap method [20] to determine the associated statistical uncertainty. For non-normal distributions, these methods give more reliable measures than the mean and standard error.

Fluctuations in the ambient magnetic field of the laboratory inevitably have some component that is, by chance, synchronous with the switching pattern of $E$. This contributes a little to the noise in the EDM, as shown in figure 4, though not to the long-time average value. We 
suppress this excess noise by correcting the EDM, block by block, according to the magnetic field readings of a magnetometer (Methods). The central value and statistical uncertainty of this magnetic field correction are given in table 1 . The correction has a negligible effect on the central value of the EDM but reduces the statistical error by $3.5 \%$.

We find that the phase of the interferometer shifts linearly with the detunings of the rf pulses at a rate of $(283 \pm 6) \times 10^{-9} \mathrm{rad} / \mathrm{Hz}$ for the first rf pulse, and $(-94 \pm 5) \times 10^{-9} \mathrm{rad} / \mathrm{Hz}$ for the second rf pulse. If the magnitude of the electric field changes when $E$ is reversed, then through the Stark shift, the rf transition frequency changes. This results in a change in the interferometer phase that correlates with $E$, mimicking the EDM phase. This systematic error can be corrected using the information contained in every block of data. The phase change resulting from a detuning of the first rf pulse is measured by $\{\mathrm{RF} 1 \mathrm{~F} \cdot B\}$, and the change in the detuning resulting from the change in electric field magnitude is measured by $\{\mathrm{RF} 1 \mathrm{~F} \cdot E\}$. The product of these two channels, together with a calibration factor which we have measured, determines the EDM-like phase due to the $E$-correlated detuning of the first rf transition, and we use this to apply a correction to each block of data. A similar correction is made for the second rf pulse. The central values and statistical uncertainties of the two rf phase corrections are given in table 1 . As an additional check, we have made measurements where we deliberately change the rf frequency when we switch $E$. We see that the resulting systematic error is entirely removed once the corrections are applied to these data, verifying the correction procedure.

There are several sources of systematic uncertainty on the EDM measurement that must 
be considered. First, there may be systematic effects, other than the rf-induced phases described above, caused by a change in field magnitude when $E$ reverses. We investigate this by changing the field magnitude intentionally by $\delta E$ when the field switches. Once the rf phase corrections are applied to these data, we find no evidence of any residual systematic EDM that depends on $\delta E$. The upper bound on the gradient of any such systematic, with respect to $\delta E$, is $-11 \times 10^{-28}(e \cdot \mathrm{cm}) /(\mathrm{V} / \mathrm{cm})$. In the $\mathrm{rf}$ regions we measure asymmetries $\delta E$ of approximately $100 \mathrm{mV} / \mathrm{cm}$ and we take this to be typical throughout the interaction region. Combining this level of asymmetry with the worst-case slope above gives a systematic uncertainty of $1.1 \times 10^{-28} e \cdot \mathrm{cm}($ table 1$)$.

Electric-field-plate potentials that are not symmetric around ground are another possible source of systematic error. We characterise this in terms of the mean potential $\bar{V}$ of the two electric field plates relative to the surrounding grounded apparatus. Near the edges of the plates, the field does not point entirely along $\hat{z}$, but the direction of the field reverses perfectly as long as $\bar{V}=0$. However, when $\bar{V} \neq 0$ the reversal is imperfect, and this, coupled with other imperfections, may result in a systematic error. We investigate this by deliberately applying large mean potentials of $\bar{V}=-1000.5 \mathrm{~V}$ and $\bar{V}=+1015.0 \mathrm{~V}$, and we find from this data a systematic shift with a slope of $(0.099 \pm 0.016) \times 10^{-28} e \cdot \mathrm{cm} / \mathrm{V}$. The plate potentials used for our dataset are measured to have a mean voltage of less than $1 \mathrm{~V}$. This results in a systematic uncertainty of $0.1 \times 10^{-28} e \cdot \mathrm{cm}$.

A study of the data taken at non-zero $\bar{V}$ revealed an unexplained correlation between the measured EDM and the frequency detuning of the first rf pulse. Unlike the effect described 
above, this systematic effect does not depend on $\delta E$. We see no evidence of the effect in the data taken at $\bar{V}=0$. Nonetheless, by considering the worst case correlation consistent with the $\bar{V}=0$ data, and the measured average frequency detuning of the first rf pulse, we calculate a conservative systematic uncertainty of $1 \times 10^{-28} e \cdot \mathrm{cm}$.

The direction of the electric field in the rest frame of the molecules rotates slightly as they move through the apparatus. This induces a geometric interferometer phase that can result in a systematic error [21]. We calculate an upper limit on this effect (see Supplementary Information) of $3 \times 10^{-30} e \cdot \mathrm{cm}$.

Magnetic fields generated inside the magnetic shields that reverse with the electric field are a potential source of systematic error. These magnetic fields are not sensed well by the magnetometers, which are outside the inner layer of magnetic shielding. We consider the three mechanisms that could generate such fields:

(i) Leakage current to the high voltage plates. The current flowing to or from each electric field plate is monitored [22] throughout the experiment. The component that reverses synchronously with $E$ is less than $1 \mathrm{nA}$ averaged over the EDM data set. A most conservative estimate (see Supplementary Information) of the possible false EDM given by these currents is $0.2 \times 10^{-28} \mathrm{e} \cdot \mathrm{cm}$.

(ii) Inner shield magnetization. It is possible that the plate-charging currents magnetize the shields, generating a magnetic field that reverses with $E$. We have determined this field by pulsing a hundred times the normal current through a similar shield set-up on the bench and measuring the resulting field with a fluxgate magnetometer. We deduce that the false EDM 
due to shield magnetization is $(-0.16 \pm 0.17) \times 10^{-28} e \cdot \mathrm{cm}$. Since this is consistent with zero, we do not make any correction to the measured EDM, but allow a systematic uncertainty of $0.25 \times 10^{-28} e \cdot \mathrm{cm}$.

(iii) Motional magnetic field. The lab-frame electric field has a magnetic component in the rest frame of the molecules $\mathbf{B}_{m}=\mathbf{E} \times \mathbf{v} / c^{2}$, which can produce a false EDM if there is also a stray magnetic field $B_{y}$. This was a limiting systematic error in [7]. The effect is strongly suppressed in our case because of the large $(8 \mathrm{MHz})$ tensor Stark splitting of the $F=1$ manifold, which renders the molecule insensitive to magnetic fields in the $x y$ plane, as discussed in [8]. Our stray $B_{y}$ is everywhere less than $30 \mathrm{nT}$, which gives a calculated false EDM of less than $5 \times 10^{-32} e \cdot \mathrm{cm}$. We have also checked empirically that the addition of a $500 \mathrm{nT}$ transverse field produces no evident effect.

A number of other consistency checks and searches for systematic errors were made and are described in detail in the Supplementary Information.

In addition to the computer-controlled switches, we make three manual reversals. The high voltage connections are swapped to reverse $E$, the magnet wires are interchanged to reverse $B$ and the $\mathrm{rf}$ cables are swapped to reverse the direction of rf propagation along the field plates. These manual changes are made infrequently - typically one switch per day - and they are valuable in identifying and eliminating systematic effects. Roughly equal numbers of blocks are taken in all eight of the manual states. When we divide the data according to these manual-reversal states and analyze each dataset separately, the EDMs obtained are consistent with one another as shown in figure 5. We also divide the data according to the 
polarization angles of the pump and probe and find no correlation with either of these.

Combining the systematic uncertainties in quadrature yields the final result $d_{e}=(-2.4 \pm$ $\left.5.7_{\text {stat }} \pm 1.5_{\text {syst }}\right) \times 10^{-28} e \cdot \mathrm{cm}$ where the first uncertainty is statistical $(68 \%$ symmetric confidence interval [23]) and the second systematic. This is consistent with zero and with the previous best measurement [7]. The result is 54 times more precise than our previous measurement [8]. Treating the statistical and systematic errors on equal terms, we can extract an upper bound on the size of the EDM, $\left|d_{e}\right|<10.5 \times 10^{-28} e \cdot \mathrm{cm}$ with $90 \%$ confidence. This is 1.5 times smaller than the previous upper limit [7].

Our error is dominated by the statistical uncertainty of the measurement. The limiting systematic errors in the measurement are sufficiently well understood that we can readily reduce them to the $10^{-29} e \cdot \mathrm{cm}$ range. Our experiment leads the way in the application of cold molecule techniques to precision measurement and we are well placed to take advantage of recent advances in the preparation [24, 25, 26] and control [27] of cold molecules to improve our measurement precision. This will allow us to probe for new particle physics at tens of $\mathrm{TeV}$.

\section{Methods summary}

Pulses of YbF are emitted by the source [17] every $40 \mathrm{~ms}$ and travel through the magneticallyshielded apparatus (figure 1) at a speed of $590 \mathrm{~m} / \mathrm{s}$. The pump detector depletes and detects the $F=1$ population while the probe detector measures the $F=0$ population. Two rf 
$\pi$-pulses, separated by the free-evolution time $T$, and tuned to the Stark-shifted hyperfine interval near $170 \mathrm{MHz}$, coherently transfer population between the $F=0$ and $F=1$ states. The primary signal is the detected $F=0$ population, which is proportional to $\cos ^{2} \phi$. The electron EDM is obtained from the part of $\phi$ that correlates with the sign of $E$, which in turn is obtained from the signal correlating with the sign of $E \cdot B$.

To measure this correlation, and a rich set of other signal correlations, the machine is put into a new state between each beam pulse. There are 9 switched parameters, hence 512 different switch combinations, and each is set 8 times in every data block (a group of 4096 pulses). For each block, the switching sequence is chosen at random from a set of possible sequences; all of these switch $B$ frequently to eliminate magnetic field noise, switch $E$ infrequently to minimize the dead time associated with this switch, and switch $E \cdot B$ aperiodically to eliminate signal drifts from this channel [29]. Between one block and the next, the relative phase of the two rf pulses is randomly changed, the linear polarizations of pump and probe are randomly rotated, and the central values of the magnetic field, the laser frequency, and the frequencies and amplitudes of the two rf pulses, are adjusted toward their ideal values.

Diagnostic data is obtained from a fluxgate magnetometer placed between the two shields, three other magnetometers around the lab, and two ammeters [22] that measure the currents flowing to the electric field plates. 


\section{Methods}

Apparatus. The apparatus is shown in Figure 1. A solenoid valve opens every $40 \mathrm{~ms}$ to release a pulse of Ar containing $2 \% \mathrm{SF}_{6}$. Ytterbium atoms laser-ablated from a target beside the valve react with the gas pulse to form YbF. The gas expands, cools, and is skimmed to form a beam with a temperature of $3 \mathrm{~K}$ and a centre-of-mass velocity of $590 \mathrm{~m} / \mathrm{s}$ [17]. The $\mathrm{YbF}$ molecules are mainly in the electronic and vibrational ground state $X^{2} \Sigma^{+}(v=0)$. Those in the rotational ground state are distributed over the hyperfine levels $F=0$ and $F=$ 1, separated by $170.254 \mathrm{MHz}$. A single-mode cw dye laser provides the linearly polarized pump and probe beams shown in figure 1 . The pump and probe are respectively tuned to the $F=1$ and $F=0$ components of the $\mathrm{A}-\mathrm{X} \mathrm{Q}(0)$ transition, so that the pump empties out the $F=1$ population and the probe measures the $F=0$ population by laser-induced fluorescence detection. Each packet of molecules passing through the probe beam generates a current pulse in the photomultiplier corresponding to $\sim 5000$ detected photons. The current pulse is digitized in 80 bins over $800 \mu$ s to produce signals such as that shown in figure 2 . The pump fluorescence is recorded in a similar way. We also record the intensities of both laser beams. The timing of the experiment is phase-locked to the mains electrical supply.

The field plates are gold-coated cast aluminium, $75 \mathrm{~cm}$ long, $7 \mathrm{~cm}$ wide, and $1.2 \mathrm{~cm}$ apart. The static electric and magnetic fields between these plates are typically $E= \pm 10 \mathrm{kV} / \mathrm{cm}$ and $B= \pm 13 \mathrm{nT}$. The plate structure doubles as a TEM transmission line to propagate $170 \mathrm{MHz}$ radiation in either direction. The rf pulses are designed to be $\pi$-pulses, so that the transfer 
of population between the $\left|F, m_{F}\right\rangle=|0,0\rangle$ state and the $\frac{1}{\sqrt{2}}(|1,+1\rangle+|1,-1\rangle)$ state occurs with unit efficiency. The first $\mathrm{rf}$ pulse is applied $1.1 \mathrm{~ms}$ after the ablation pulse, when the molecules are approximately $13 \mathrm{~cm}$ inside the plates. The second $\mathrm{rf}$ pulse is applied after the free evolution time of $T=642 \mu \mathrm{s}$. Both pulses are $18 \mu \mathrm{s}$ long rf magnetic field pulses polarised along $\hat{x}$ (figure 1). If the $\pi$-pulses are imperfect, coherence between $F=0$ and $F=1$ states results in additional, unwanted interference terms. We suppress these terms by averaging the relative rf phase $\phi_{0} \pm \pi / 2$ over the $(R F \Phi)$ switch and by randomising $\phi_{0}$ between blocks. The theory of two-pulse rf transitions within this three-level manifold is developed fully in section IV.B of [28].

The beam line is enclosed by two layers of magnetic shielding. The high voltage feeds pass close together through a single hole in the inner magnetic shield near the center of the plates in order to minimize shield magnetization by the charging currents. A fluxgate magnetometer between the shields measures the magnetic field parallel to $\hat{z}$ near the probe detector. Three other magnetometers of lower sensitivity are used to monitor the laboratory magnetic field - one near the beam machine, one close to the high voltage relays that reverse $E$, and one close to the computer interface that controls the experiment. These are also read after every pulse and their primary purpose is to ensure that $E$-reversal does not generate a magnetic field. The same analog-digital converter board that reads these signals also monitors two dummy voltages, a battery and a short circuit. These are used to check that there are no systematic errors in the signal processing electronics and data analysis.

Characterizing the machine. We have mapped the spatial variation of the the electric, 
magnetic and rf fields, as described in [16]. We find that the electric field varies by roughly $1 \%$ over the length of the plates, and that the ambient magnetic field is typically less than $10 \mathrm{nT}$ throughout the region that we use for the interferometer. The rf field has a small standing wave ratio, corresponding to a $4 \%$ power reflection coefficient at each end. In the TEM mode, the rf electric field is constrained by the same boundary conditions as the static field, ensuring that the $\mathrm{rf}$ magnetic field is accurately perpendicular to $E$ and to the propagation direction. The rf field at each end of the plates has some ellipticity, due to the transient where the transmission line is coupled to coaxial cable. This decays away over a few $\mathrm{cm}$.

Switching sequence. As discussed in the main text, 9 separate parameters are switched in the experiment. A set of 4096 beam pulses forms a block of data, within which all 512 combinations of switch states are covered equally. The sequence of switches applied within a block, known as the switching pattern, must satisfy three requirements. First, the magnetic field should switch frequently to eliminate magnetic field noise. Second, the electric field must switch less often because $E$ reversal incurs a dead time of $14 \mathrm{~s}$. This allows time to discharge and recharge the plates while keeping the transient currents below $5 \mu \mathrm{A}$ to avoid magnetizing the shields. By the time we restart data acquisition the current is close to its steady value of $\sim 1 \mathrm{nA}$. This restriction is important because a magnetic field reversing with $E$ can generate a systematic error. Third, the switching sequence of $E \cdot B$ should be as aperiodic as possible so that signal drifts do not influence this channel [29]. Within these restrictions, there are still a large number of possible switching patterns from which the computer randomly chooses one at the start of every block. At the end of each block 
the channel values are calculated and some of these are used to optimize the running of the machine. For example, $\{B\}$ measures how well the operating fields are centered around $B=0$ and this provides an error signal at the end of each block that is fed back to compensate for small drifts of the ambient field. Similarly, $\{\mathrm{RF} 1 \mathrm{~F}\}$ and $\{\mathrm{RF} 2 \mathrm{~F}\}$ are used to servo the rf frequencies to resonance while $\{\mathrm{RF} 1 \mathrm{~A}\}$ and $\{\mathrm{RF} 2 \mathrm{~A}\}$ are used to servo the rf amplitudes to the $\pi$-pulse condition. The channel $\{\mathrm{LF}\}$ is used to keep the laser on resonance. Between blocks the the mean relative phase $\phi_{0}$ between the two rf pulses is randomly changed and the linear polarizations of the pump and probe laser beams are randomly rotated. Including the dead time, each block takes approximately 6 minutes to accumulate.

EDM interaction. The interaction of the electron in a molecule with an applied electric field is more complicated than that of a free electron, described in the introduction. It is possible however to write the interaction as $-\mathbf{d} \cdot \mathbf{E}_{\text {eff. }}$ The effective electric field, $\mathbf{E}_{\text {eff }}$, which depends non-linearly on the applied electric field accounts for the complexity of the molecular environment. Under our operating conditions the effective field has magnitude $14.5 \mathrm{GV} / \mathrm{cm}$ and is aligned antiparallel to the applied field $[10,11,12,13,14,15]$. Thus, the energy shift of the $\left(F=1, m_{F}\right)$ state of the molecule due to the electron EDM is $-d_{e} E_{\text {eff }} m_{F}$ where $E_{\text {eff }}=-14.5 \mathrm{GV} / \mathrm{cm}$. In deriving the EDM we have assumed that the effective field is known exactly. While there is some uncertainty in the theoretical calculation, even an uncertainty of $10 \%$ would have no impact on our error at the level reported in this Letter. 


\section{References}

[1] I. B. Khriplovich, S. K. Lamoreaux, CP Violation Without Strangeness (Springer, New York, 1997).

[2] M. Pospelov, A. Ritz, Electric dipole moments as probes of new physics Annals Phys. 318 119-169, (2005).

[3] E. D. Commins, Electric dipole moments of leptons, in Advances in Atomic, Molecular, and Optical Physics, Vol. 40, B. Bederson and H. Walther (Eds.), Academic Press, New York, pp. 1-56 (1999).

[4] E. D. Commins and D. DeMille, "The electric dipole moment of the electron", Chapter 14 in Lepton Dipole Moments Eds. B. L. Roberts and W. J. Marciano, (World Scientific, Singapore 2010).

[5] A. D. Sakharov, Violation of CP invariance, C asymmetry, and baryon asymmetry of the universe, Pis'ma ZhETF 5, 32-35 (1967). [Sov. Phys. JETP Lett. 5, 24-27 (1967).]

[6] A. R. Edmonds, Angular Momentum in Quantum Mechanics, (Princeton University Press, 1996).

[7] B. C. Regan, E. D. Commins, C. J. Schmidt, D. DeMille, New Limit on the Electron Electric Dipole Moment, Phys. Rev. Lett 88, 071805 (2002). 
[8] J. J. Hudson, B. E. Sauer, M. R. Tarbutt, E. A. Hinds, Measurement of the Electron Electric Dipole Moment Using YbF Molecules, Phys. Rev. Lett. 89, 023003 (2002).

[9] E. A. Hinds, Testing time reversal symmetry using molecules, Physica Scripta T70, 3441 (1997).

[10] M. G. Kozlov, V. F. Ezhov, Enhancement of the electric dipole moment of the electron in the YbF molecule, Phys. Rev. A 49, $4502-4507$ (1994)

[11] M. G. Kozlov, Enhancement of the electric dipole moment of the electron in the YbF molecule , J. Phys. B 30, L607-L612 (1997)

[12] A. Titov, M. Mosyagin, V. Ezhov, P,T-Odd Spin-Rotational Hamiltonian for YbF Molecule, Phys. Rev. Lett. 77, 5346-5349 (1996)

[13] H. M. Quiney, H. Skaane, I. P. Grant, Hyperfine and PT-odd effects in $Y b F^{2} \Sigma$, J. Phys. B 31, L85-L95 (1998)

[14] F. A. Parpia, Ab initio calculation of the enhancement of the electric dipole moment of an electron in the YbF molecule, J. Phys. B 31, 1409-1430 (1998)

[15] N. Mosyagin, M. Kozlov, A. Titov, Electric dipole moment of the electron in the YbF molecule, J. Phys. B 31, L763-L767 (1998). 
[16] J. J. Hudson, H. T. Ashworth, D. M. Kara, M. R. Tarbutt, B. E. Sauer, E. A. Hinds, Pulsed beams as field probes for precision measurement, Phys. Rev. A. 76, 033410 (2007).

[17] M. R. Tarbutt, B. E. Sauer, J. J. Hudson, E. A. Hinds, V. A. Ryzhov, V. L. Ryabov, V. F. Ezhov, A jet beam source of cold YbF radicals, J. Phys. B 35, 5013-5022 (2002).

[18] T. Ma, C. Butler, J. M. Brown, C. Linton, T. C. Steimle, Optical Zeeman Spectroscopy of Ytterbium Monofluoride, YbF, J. Phys. Chem. A 113, 8038-8044 (2009).

[19] R.A. Maronna, D.R. Martin, V.J. Yohai, Robust statistics; theory and methods, (Wiley, Chichester, 2006).

[20] B. Efron, R. Tibshirani, Bootstrap Methods for Standard Errors, Confidence Intervals, and Other Measures of Statistical Accuracy, Stat. Sci. 1, 54-75 (1986).

[21] M. R. Tarbutt, J. J. Hudson, B. E. Sauer, E. A. Hinds, Prospects for measuring the electric dipole moment of the electron using electrically trapped polar molecules, Faraday Discuss, 142, 37-56 (2009).

[22] B. E. Sauer, D. M. Kara, J. J. Hudson, M. R. Tarbutt, E. A. Hinds, A robust floating nanoammeter, Rev. Sci. Instr. 79, 126102 (2008).

[23] B. Efron, Better bootstrap confidence intervals, J. Am. Stat. Assoc. 82, 171-185 (1987). 
[24] S. M. Skoff, R. J. Hendricks, C. D. J. Sinclair, J. J. Hudson, D. M. Segal, B. E. Sauer, E. A. Hinds, M. R. Tarbutt, Diffusion, thermalization and optical pumping of YbF molecules in a cold buffer gas cell, Phys. Rev. A 83, 023418 (2011).

[25] J.F. Barry, E.S. Shuman, D. DeMille, A Bright, Slow Cryogenic Molecular Beam Source for Free Radicals, arXiv:1101.4229v1 [physics.atom-ph] (2011).

[26] N. R. Hutzler, M. Parsons, Y. V. Gurevich, P. W. Hess, E. Petrik, B. Spaun, A. C. Vutha, D. DeMille, G. Gabrielse, J. M. Doyle, A cryogenic beam of refractory, chemically reactive molecules with expansion cooling, arXiv:1101.4217v1 [physics.atom-ph] (2011)

[27] S. Y. T. van de Meerakker, H. L. Bethlem and G. Meijer, "Slowing, trapping and storing of polar molecules by means of electric fields", Chapter 14 in Cold Molecules: theory, experiment, applications Eds. R. Krems, W. Stwalley and B. Friedrich, (CRC Press, Taylor and Francis, Boca Raton 2009).

[28] M. R. Tarbutt, J. J. Hudson, B. E. Sauer, E. A. Hinds, Chapter 15 in Cold Molecules: theory, experiment, applications Eds. R. Krems, W. Stwalley and B. Friedrich, (CRC Press, Taylor and Francis, Boca Raton 2009).

[29] G. E. Harrison, M. A. Player, P. G. H. Sandars. A multichannel phase-sensitive detection method using orthogonal square waveforms. J. Phys. E 4, 750-754 (1971). 


\section{Acknowledgments}

We acknowledge the contributions of Paul Condylis and Henry Ashworth. We are grateful for technical assistance from Jon Dyne and Valerijus Gerulis. This work was supported by the UK research councils STFC and EPSRC, and by the Royal Society. J.J.H. is supported by an STFC Advanced Fellowship.

\section{Author contributions}

J. J. H. was involved in all aspects of the measurement, led the analysis, and drafted the manuscript. D. M. K. developed many of the systematic tests, worked on taking the dataset, and contributed to the analysis. I. J. S. had primary responsibility for taking the dataset, and contributed to the development of the data acquisition techniques. B. E. S. was involved in all aspects of the measurement, and designed much of the hardware. M. R. T. built the molecular beam source, contributed to the analysis, and drafted the manuscript. E. A. H. contributed to the analysis, drafted the manuscript, and led the team. All authors were equally involved in setting the direction of the work, discussed the results, and improved the manuscript.

\section{Author information}

Reprints and permissions information is available at www.nature.com/reprints. Correspondence and requests for materials should be addressed to e.a.hinds@imperial.ac.uk . 


\begin{tabular}{lccc}
\hline \hline & Correction & Stat. & Syst. \\
\hline Magnetic field correction & -0.3 & 1.7 & $<0.1$ \\
rf1 phase correction & 5.0 & 0.9 & $<0.1$ \\
rf2 phase correction & 0.5 & 0.7 & $<0.01$ \\
\hline Uncorrected $\delta E$ effects & - & - & 1.1 \\
$\bar{V}$ uncertainty & - & - & 0.1 \\
$\{$ RF1F $\}$ correlation & - & - & 1.0 \\
Geometric phase & - & - & 0.03 \\
Leakage currents & - & - & 0.2 \\
Shield magnetization & - & - & 0.25 \\
$v \times E$ effect & - & - & 0.0005 \\
\hline \hline
\end{tabular}

Table 1: Summary of applied corrections and uncorrected systematic uncertainties. The units are $10^{-28} \mathrm{e} \cdot \mathrm{cm}$. The statistical uncertainty on the corrections gives a measure of their random spread over the whole dataset. In the final analysis the corrections are applied block-byblock, so these statistical uncertainties are naturally incorporated in the final EDM statistical uncertainty. The systematic uncertainty in the corrections is negligible. 


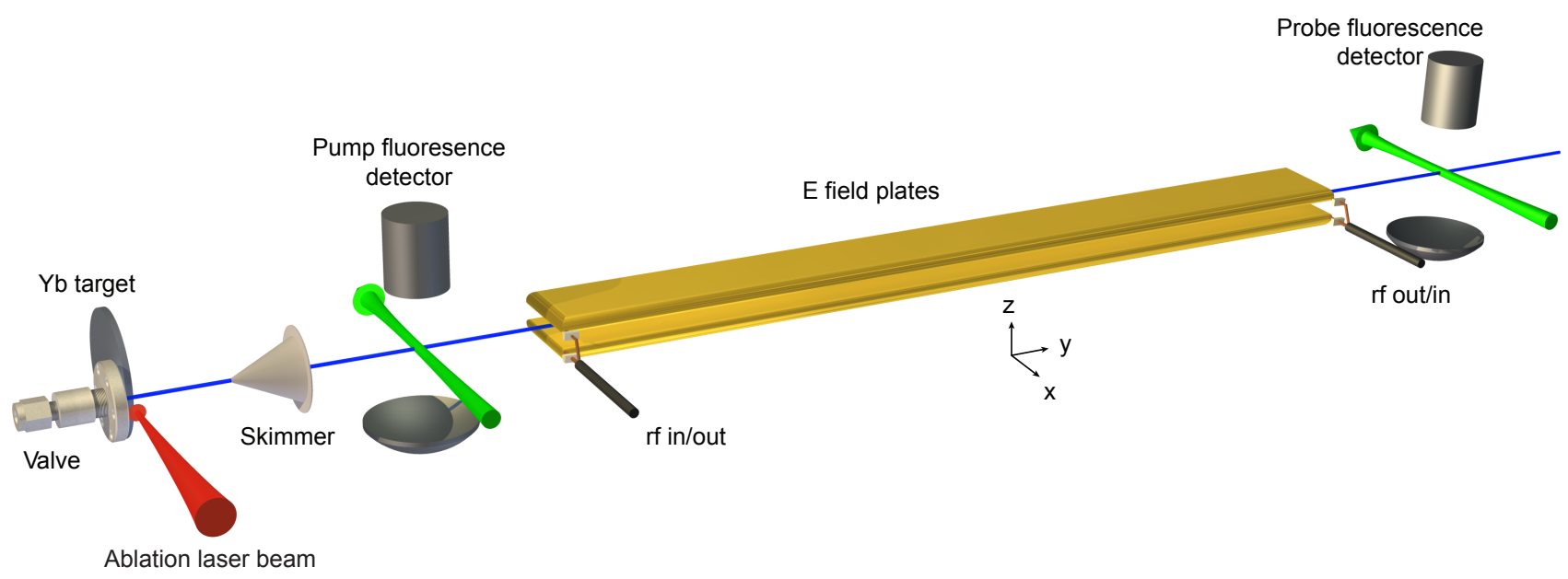

Figure 1: Schematic diagram of the pulsed molecular beam apparatus.

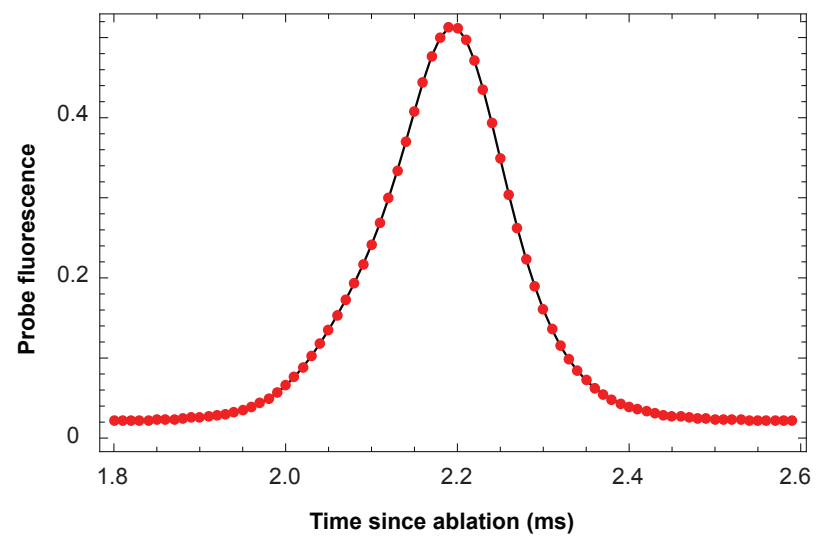

Figure 2: Fluorescence from a typical beam pulse, measured on the probe detector. 


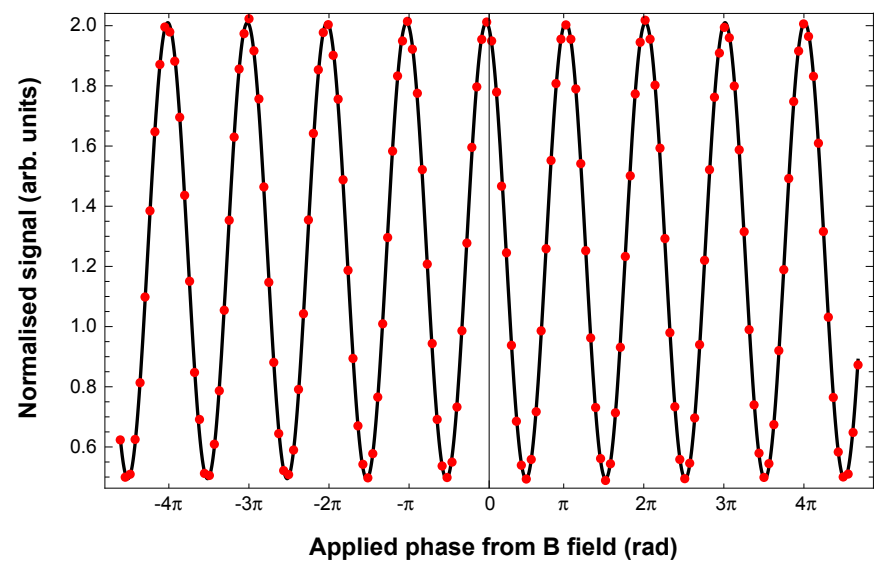

Figure 3: Interferometer fringes produced by magnetic field scan. Dots: probe fluorescence normalized to pump fluorescence. Line: fit to cosine-squared model.

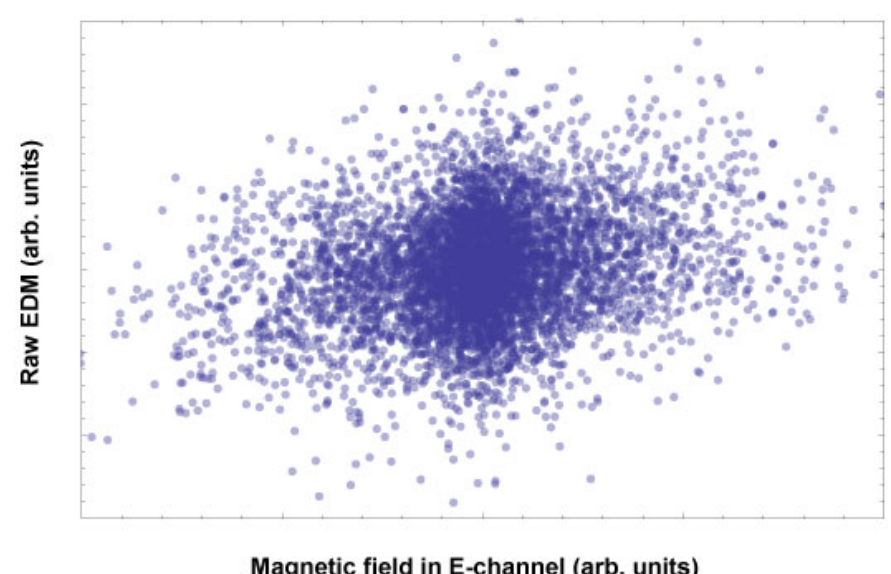

Figure 4: The magnetic field correlated with the E reversal, measured at the fluxgate magnetometer, versus the EDM values. A slope is evident. The majority of measurements are not significantly perturbed by the magnetic field, but a small fraction do benefit from correction. 


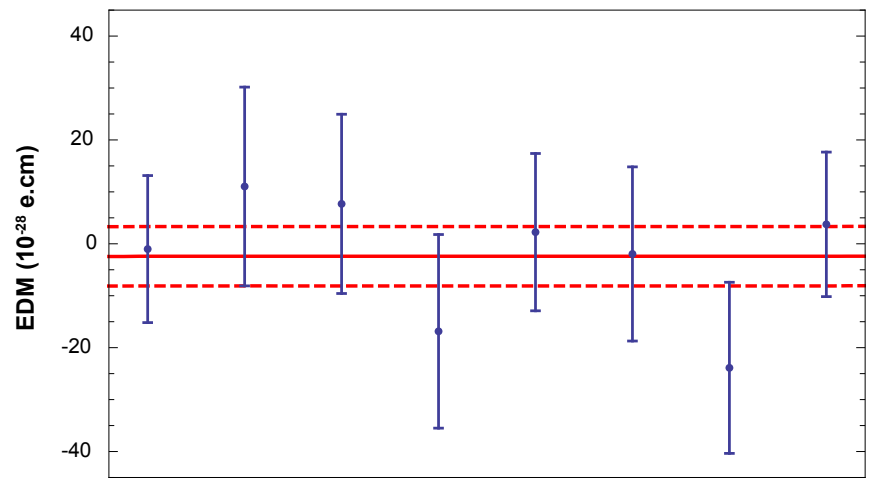

Figure 5: EDM values for each manual-reversal state of the machine. The error bars indicate the $68 \%$ confidence level. The most important manual-reversal is the electric-field reversal: the first four points correspond to one electric field configuration, and the last four the other. The solid and dashed lines show the mean value and its statistical error. 


\section{Supplementary information}

Here we present further details of our calculations of systematic EDM limits, and describe the additional tests and consistency checks that we applied to the dataset.

\section{Geometric phase}

We calculate a worst case example of the edm from a geometric phase. The geometric phase is equal to the solid angle subtended by the rotation of the electric field [21]. Our electric field map [16] (Method) determines how the plate spacing varies along the beam direction $y$, from which we know that the field rotates around the $x$ axis (figure 1) by less than $\pm 0.5 \mathrm{mrad}$. From the geometry of the plates, we expect a similar field rotation around the $y$ axis. However, these rotations do not change sense when the electric field is reversed and therefore do not generate a systematic error. To result in an apparent EDM phase they must work in concert with a rotation that does change sense when $E$ is reversed. Such a rotation would result for example from patch fields due to local variation of the composition, crystalline structure, or contaminants on the plate surfaces. As a worst case, we imagine a $1 \mathrm{~V}$ rectangular patch filling the second half of the interferometer and covering half the width of one plate. In the rest frame of the molecules this sweeps out a solid angle whose sign reverses when the applied field is reversed, causing a systematic error. After averaging over the molecular beam trajectories we calculate that the false EDM in this example is $3 \times 10^{-30} e \cdot \mathrm{cm}$, which we take in table 1 as an upper limit. 


\section{Leakage currents}

Let us assume that the $1 \mathrm{nA}$ average leakage current somehow manages to flow up the edge of one plate and down the opposite edge of the other over half the full length of the plates. Over the path of the molecules, this generates a mean $B_{z}$ field of $5 \mathrm{fT}$. We take the corresponding false EDM, $\left|\mu_{B} B_{z} / E_{\text {eff }}\right|=0.2 \times 10^{-28} e \cdot \mathrm{cm}$, as the upper limit of this effect and treat it in table 1 as an additional uncertainty.

\section{Other systematic checks}

The 511 channels other than $\{E \cdot B\}$ have been examined to ensure that the experiment operates correctly. Most are expected to be consistent with zero and we find that they are. A false EDM is generated if, for any combination $X$ of the switched parameters, there are channels $\{X \cdot B\}$ and $\{X \cdot E\}$ that are both non-zero. Searching all channels, we find that only RF1F and RF2F have this property, and we have already corrected for these in the analysis described in the main text.

We also analyse the early- and late-arriving halves of the probe pulses separately, and find that the EDMs derived from each are consistent.

As well as the main data from the probe detector, we analyze the data from the pump detector, a magnetometer between the shields, three magnetometers placed around the lab, two leakage current monitors, a battery dummy-input and a short-circuit dummy-input (Methods). We use the same analysis routines, searching for signals that correlate with any of the switches. From all the channels of all these detectors, we find only three that are expected to 
be consistent with zero but are not. The magnetometer between the shields registers a small magnetic field that correlates with the state of the rf-phase switcher, showing that the field generated by this device is different in its two states. This field is too small to be of concern and in any case does not depend on $E$. The magnetometer that is close to the electric field relay measures a magnetic field that correlates with $E$, but none of the other magnetometers register this field showing that it falls off too rapidly with distance to have any significant effect on the molecules (this fact is also shown by the first line of table 1). The two leakage current monitors register a small signal correlated with $B$, but this is much too small to be of concern. 